\title{
Assessment of the Transmission Risk of Schistosomiasis after Flooding - North Poyang Lake, Jiangxi Province, China, 2020
}

\author{
Shan $\mathrm{Lv}^{1, *}$; Fan Yang'; Zhiqiang Qin ${ }^{1}$; Chunli Cao ${ }^{1}$; Jing Xu' ${ }^{1}$; Shizhu $\mathrm{Li}^{1}$; Xiao-nong Zhou
}

\section{Summary}

What is already known about this topic?

Over $90 \%$ of Oncomelania snails, the only intermediate host of Schistosoma japonicum, are distributed in the middle and low reaches of Yangtze River. Flooding can extend the distribution of Oncomelania snails and hence accelerate the transmission of schistosomiasis.

What is added by this report?

Although the dispersal of Oncomelania snails was negligible in north Poyang Lake after flooding in 2020,

2 samples of cattle feces with Schistosoma egg and 2 infected snails samples were indeed found. All four risk sites were distributed in Lushan County. Cattle feces were observed in the six out of seven field sites in Lushan County.

What are the implications for public health practice?

The present national control strategy focusing on control of infection source should be reinforced in Lushan and other schistosomiasis endemic areas. Overlaps of infected snails and cattle feces with Schistosoma egg were not observed, which called for intensive surveillance in Lushan County.

The Oncomelania hupensis is the only intermediate host of Schistosoma japonicum (S. japonicum) in China. Approximately $97.36 \%$ habitats infested by Oncomelania snails are in the middle and low reaches of the Yangtze River (1). The typical habitat of Oncomelania snails is marshland along the Yangtze River, its major branches, and the connected lakes (2). Such habitats are submerged in water in summer and emerge in winter. Therefore, rainfall and flooding impact the distribution of snails and the transmission dynamics of schistosomiasis (3). Acute schistosomiasis was frequently observed in patients who had contact with water due to rescue work in flooding (4). The water level hit a historical high at many hydrological stations in middle and lower reaches of Yangtze River in the summer of 2020. The most affected areas were in Jiangxi and Anhui provinces, which were at high risk of schistosomiasis transmission (1). A risk assessment in Jiangxi was conducted for schistosomiasis transmission and provided interventions after flooding. Human stool samples, Oncomelania snails, and animal feces released to the environment were collected in two counties, i.e., Lushan and Lianxi. Although no infected humans were found in the survey, infections in snails and animal feces were discovered from four field sites in Lushan. Our results indicated the intensive surveillance should be implemented post-flooding.

Two neighboring counties, i.e., Lushan and Lianxi, located in the northwest bank of Poyang Lake leading to Yangtze River were selected. The evidence showed that the density of Oncomelania snails increased significantly and exceeded that in the south lake according to survey in 2016 (5). The area drew attention because the only human acute schistosomiasis case and infected cattle case in 2019 was reported in this area (6). Two townships in each county were selected and one village was chosen in each township. About 100 residents who reported contact with water during flooding and another 100 participants without record of water contact in each village were randomly selected in the study. If there was no adequate eligible participant in the village, other potential individuals from neighboring villages in the same township were included. Stool samples of over $90 \mathrm{~g}$ per individual were collected for triple detection of $S$. japonicum miracidia hatching after nylon mesh bag concentration (7). At least three extant habitats of Oncomelania snails and suspected environments impacted by flooding were selected for the snail survey. More than 500 sampling frames $\left(0.11 \mathrm{~m}^{2}\right)$ were set for screening Oncomelania snails in each habitat or environment, and over 1,000 snails were collected. Every 50 snail specimens were pooled for DNA extraction and detected for $S$. japonicum by loop-mediated isothermal amplification (LAMP) (8). The animal feces released in the surveyed snail habitats were collected. At least 20 samples (all if less than 20) were collected in each habitat. Over $150 \mathrm{~g}$ per sample was collected for triple detection of $S$. japonicum using a miracidial hatching 
test with a plastic tube. The potential host animals of stool samples were recorded.

The sentinel-1A radar image was characterized around July 12, 2020, when Xingzi hydrological station in Lushan County experienced the highest water level and on October 19 when the present field survey was commenced. The water body was extracted from the image in ENVI 5.1 (Exelis Visual Information Solutions, Boulder CO, USA) and mapped in ArcGIS 10.1 (ESRI, Redlands CA, USA). The images in the same period in 2018 and 2019 were also collected to compare the water coverage between 2020 and the previous 2 years. The test statistic for the occurrence rate of Oncomelania snails in sampling frames, the LAMP-positive rate of snails, and overall schistosomiasis prevalence in animal feces samples in the 2 counties were performed in IBM SPSS Statistics 19.0 (IBM Corp; Armonk NY, USA).

A total of 452 and 403 stool samples from individuals from Lianxi and Lushan counties, respectively, were collected for the survey. No infection was detected in all 5 villages. A total of 19 field sites were visited during the survey, and 11 sites were infested by Oncomelania snails. The mean snail density was 0.09 per frame $\left(0.11 \mathrm{~m}^{2}\right)$ with a range between 0.01 and 0.28 . The occurrence rate of Oncomelania snails in sampling frames was $10.17 \%$ in Lushan, which was higher than the $4.89 \%$ found in Lianxi, in which the difference was statistically significant $(P<0.05)$. A total of 11 and 10 pooled samples of snails were detected for Schistosoma by LAMP in Lushan and Lianxi, respectively, in which only 2 samples from Lushan County were positive. A total of 71 animalfeces samples, including 46 cattle samples, 10 sheep samples, 7 dogs samples, and 8 unknown samples, were collected from 8 visiting sites. All cattle samples were collected from 6 out of 7 visiting sites in Lushan County in which 2 cattle samples were miracidial hatching positive from separated sites located in Jiaotang Township (Figure 1). However, no Oncomelania snail was found from the 2 positive cattle samples.

Extant snail habitats according to the national survey on Oncomelania in 2016 were mapped. Most habitats were submerged in water until late October 2020 (Figure 1). The time of flood waters receding was remarkably later than that in 2018 (Figure 2A) and 2019 (Figure 2B). The radar image showed there was only little increase in water area in the study area, except for the area located in southwestern Lushan County (Figure 3). Few breaches were occurring in major dams along Poyang Lake during the flooding, although the water level obviously increased compared to the average level between 2018 and 2019.

\section{DISCUSSION}

Although no notable snail habitats extended due to flooding in 2020, risk factors including infective cattle feces and infected snails were widely identified in middle and south researching area. These findings indicated much higher risk for schistosomiasis transmission than previously evaluated risk in annual routine assessments by the National Institute of Parasitic Diseases of China CDC. Therefore, surveillance and management of domestic animal feces must be enforced, and extensive assessment should be conducted sooner.

Cattle were the major source of infection in the study area and the national control strategy (i.e., the integrated strategy with emphasis on control of source of infection) should be enforced. Domestic mammals, particularly cattle, are playing an essential role in schistosomiasis control. A new strategy commencing in 2004 focused on controlling the source of infection after pilot studies (9). The measures include replacing farming cattle with machines, forbidding grazing on snail-infested grassland, managing human and animal feces, etc. The remarkable decrease in the prevalence of schistosomiasis in humans and animals across the country proves the effectiveness of the strategy (10).

However, cattle are reemerging in some areas. All cattle in schistosomiasis-endemic areas in Lushan County were completely removed before 2018, while the number of cattle in 2018 and 2019 were 1,063 and 1,860 , respectively, according to Annals of Statistics for Schistosomiasis Control. An infected cattle was found in 2019 during the routine surveillance. Animal feces were observed in six out of seven visiting sites and cattle feces were found in all the six sites, which indicated reemergence of cattle in Lushan County was common. In contrast, around 10 cattle in the endemic area of Lianxi County were reported annually since 2016, but no infection was found. Furthermore, cattle feces were not observed in all 12 visiting sites in the present study. Our findings demonstrated that emerging cattle challenged the achievement even at the stage of low prevalence. Therefore, control of infection sources, particularly cattle, is recommended in Lushan and other counties with the similar situation.

In the study four high-risk sites were identified surrounding the northern lake in Lushan County. 


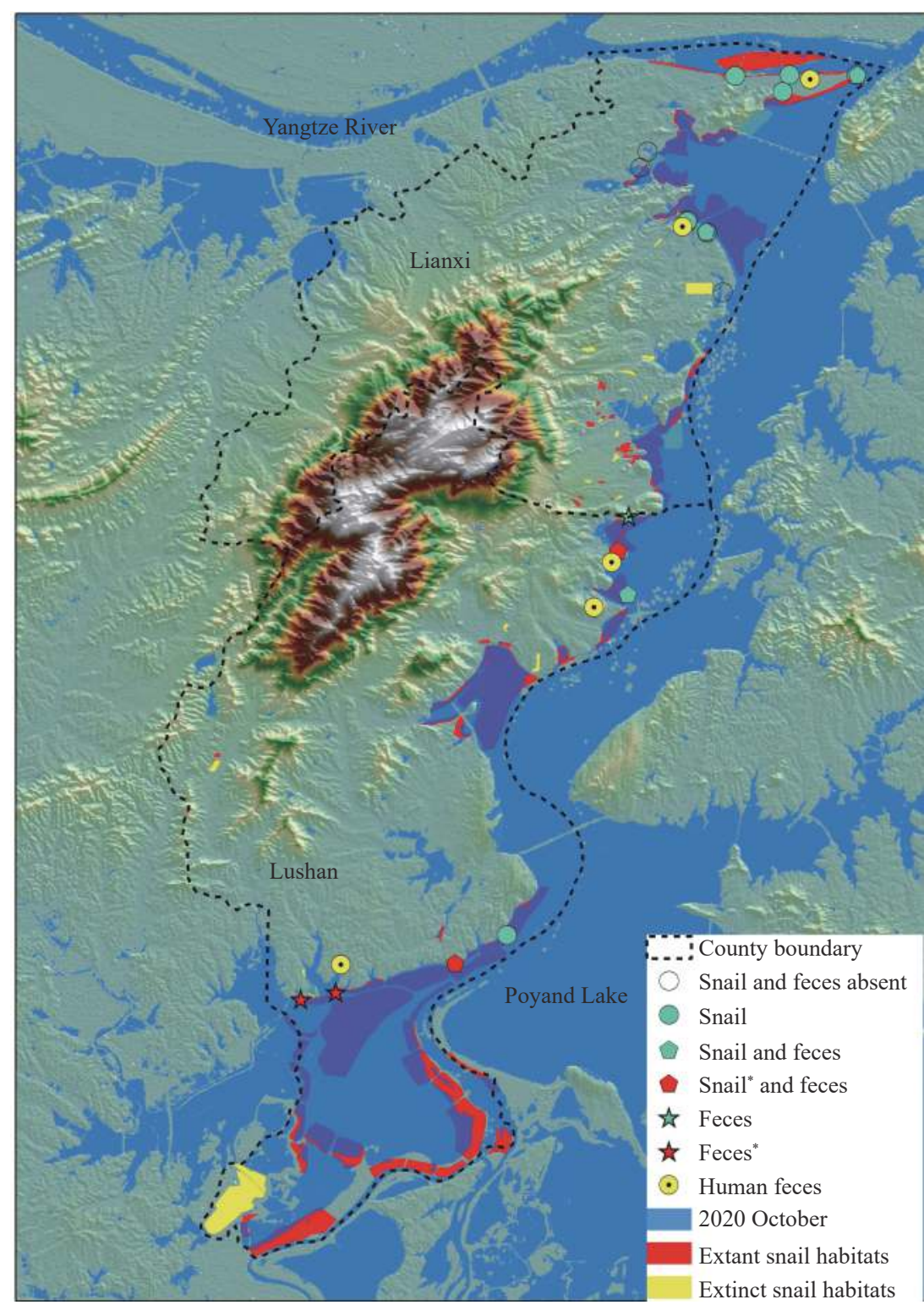

FIGURE 1. Geographical distribution of visited sites with major findings in Lianxi and Lushan counties, Jiangxi Province, China, October 2020.

Note: Snail: Oncomelania hupensis; Feces: animal feces released to environment; *: infected snails or feces with Schistosoma japonica eggs; Human feces: villages where residents' stool samples were examined for Schistosoma infection; 2020 October: water distribution in October 2020.

They were distributed both in north and south bank of the lake, which indicated a wide risk of schistosomiasis transmission in this county. In addition, no overlap was observed between the four sites, which implied the cattle were moving in a large range. Therefore, extensive surveillance for schistosomiasis transmission should be implemented.

It is well known that the flooding can carry Oncomelania snails to the new areas, and hence, the distribution of snails can extend (4). In our study region, the area covered by flooding was not significantly increased due to the relatively higher elevation of lake bank and few breaches in dams. In addition, the submergence of snail habitats is remarkably prolonged compared to that in previous years, which challenges the fall reproduction of Oncomelania snails and potentially decreases the snail density in the next spring. However, the situation in the south Poyang Lake may be different. It is worth to conducting further assessments.

This study was subjected to some limitations. Flooding in 2020 widely impacted the schistosomiasis 

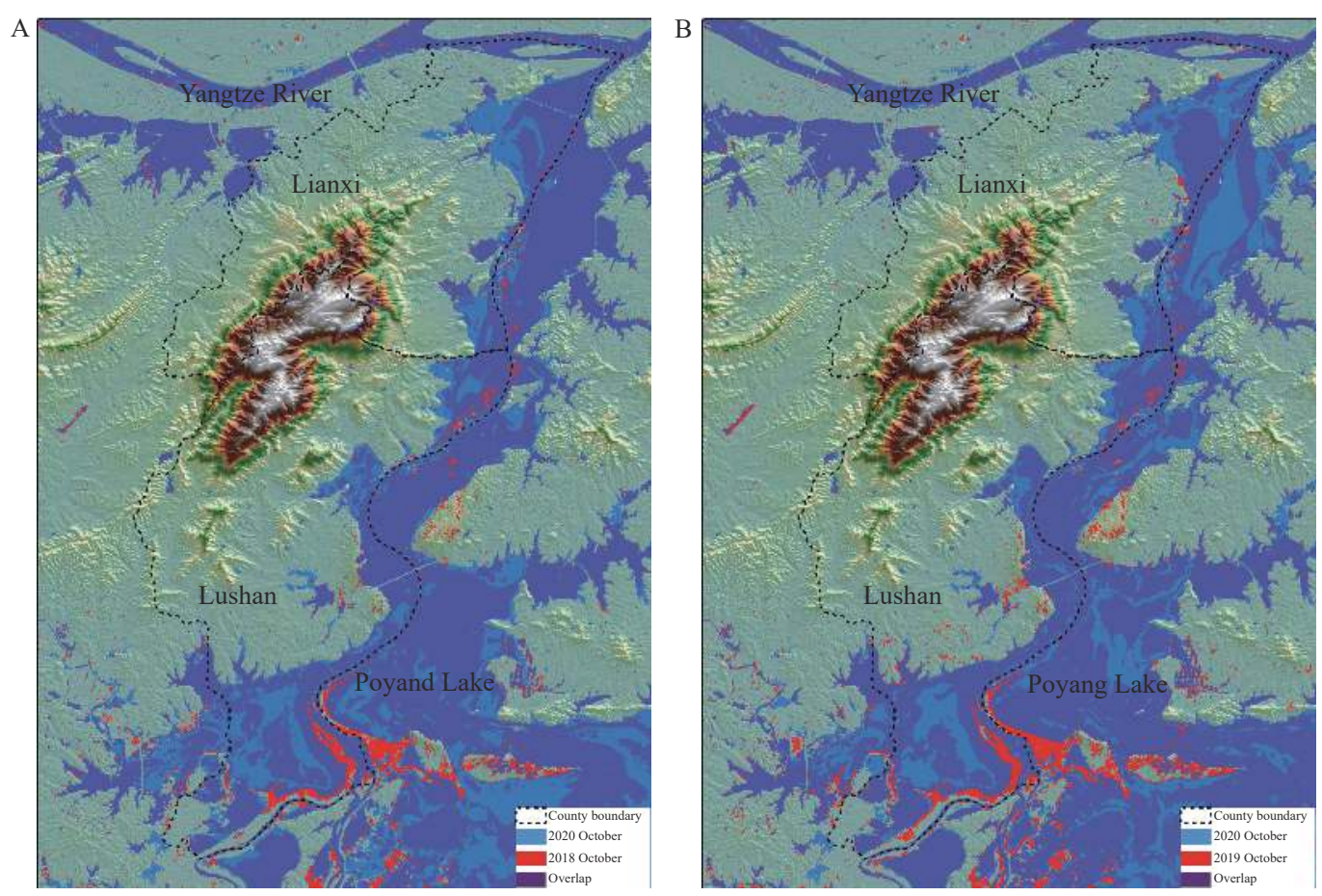

FIGURE 2. Comparison of water body distribution in north Poyang Lake during the same periods. (A) October, 2020 and October, 2018; (B) October, 2020 and October, 2019.

Note: Overlap: the overlap of water body between October, 2020 and (A) October, 2018 or (B) 2019.

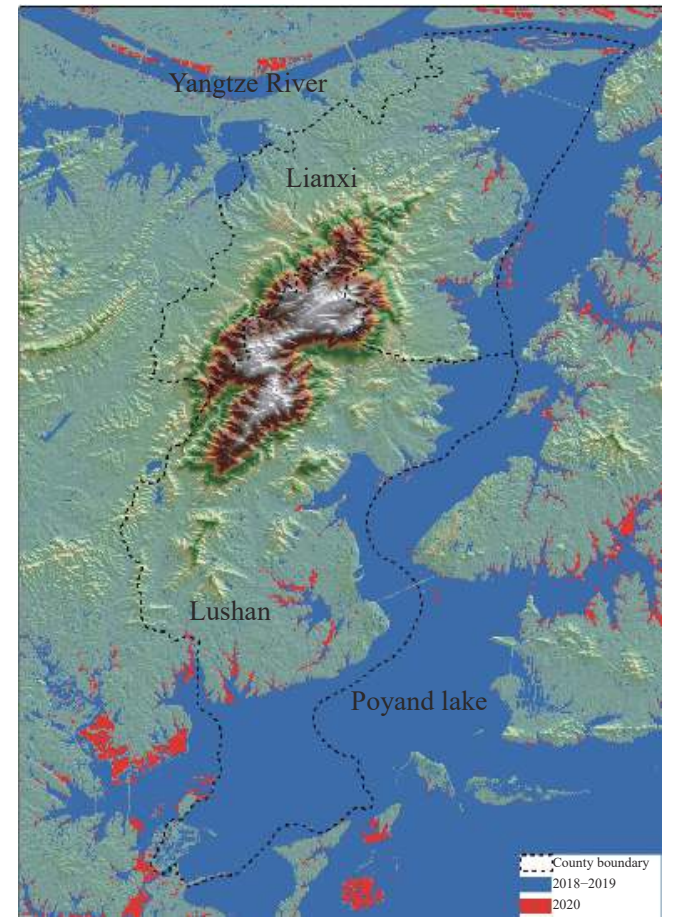

FIGURE 3. Increased area affected by flooding in 2020 compared to that in 2018 and 2019 in Lianxi and Lushan counties, Jiangxi Province, China.

Note: 2018-2019: the maximum water area at highest water levels in 2018 and 2019; 2020: the extended flooded areas during 2020 compared to 2018-2019. endemic counties, but only 2 adjacent counties on the north bank of Poyang Lake were included in this study. Although our findings may represent the situation in some endemic counties, further assessment or surveillance should be performed in other counties.

In conclusion, our findings indicated high transmission risk of schistosomiasis in some areas. Management of infection sources, particularly cattle feces, should be strengthened in the area surrounding the lake. Further assessment on impact of flooding on snail habitats in extensive area should be conducted.

Acknowledgements: Jiangxi Provincial Institute of Parasitic Diseases; Institutes of Schistosomiasis Control in Lianxi and Lushan counties; Hai Zhu from Anhui Provincial Institute of Parasitic Diseases; Feng Wang from Wuhu Institute of Endemic Disease Control; Dainian Peng from Anqing Institute of Schistosomiasis Control; Jianjun Dai and Conggui He from Guichi Institute of Schistosomiasis Control; Bin Ruan and Kai Tian from Yian Institute of Schistosomiasis Control.

Conflicts of interest: The authors declared no competing interests.

doi: $10.46234 / \mathrm{ccdcw} 2021.022$

\# Corresponding author: Shan Lv, lvshan@nipd.chinacdc.cn.

\footnotetext{
${ }^{1}$ National Institute of Parasitic Diseases, Chinese Center for Disease Control and Prevention; Chinese Center for Tropical Diseases
} 
Research; WHO Collaborating Centre for Tropical Diseases; National Center for International Research on Tropical Diseases, Ministry of Science and Technology; Key Laboratory of Parasite and Vector Biology, Ministry of Health, Shanghai, China.

Submitted: December 07, 2020; Accepted: January 27, 2021

\section{REFERENCES}

1. Zhang LJ, Xu ZM, Guo JY, Dai SM, Dang H, Lv S, et al. Endemic status of schistosomiasis in People's Republic of China in 2018. Chin J Schisto Control 2019;31(6):576 - 82. http://dx.doi.org/10.16250/ j.32.1374.2019247. (In Chinese).

2. Li ZJ, Ge J, Dai JR, Wen LY, Lin DD, Madsen H, et al. Biology and control of snail intermediate host of Schistosoma japonicum in the People's Republic of China. Adv Parasitol 2016;92:197-236. http://dx.doi.org/10.1016/bs.apar.2016.02.003.

3. Cao CL, Li SZ, Zhou XN. Impact of schistosomiasis transmission by catastrophic flood damage and emergency response in China. Chin J Schisto Control 2016;28(6):618 - 23. http://dx.doi.org/10.16250/ j.32.1374.2016215. (In Chinese).

4. Chen W, Yang XX, Huang XB, Zhang YH, Cai SX, Liu JB, et al. Influence of flood in 1998 on schistosomiasis epidemic. Chin J Schisto Control 2000; 12(4): 202-5. http://www.cnki.com.cn/Article/CJFD Total-XXCB200004004.htm. (In Chinese).
5. Hu F, Ge J, Lv SB, Li YF, Li ZJ, Yuan M, et al. Distribution pattern of the snail intermediate host of schistosomiasis japonica in the Poyang Lake region of China. Infect Dis Poverty 2019;8(1):23. http://dx.doi.org/ 10.1186/s40249-019-0534-8.

6. Zhang LJ, Xu ZM, Dang H, Li YL, Lv S, Xu J, et al. Endemic status of schistosomiasis in People's Republic of China in 2019. Chin J Schisto Control 2020;32(6):551 - 8. http://dx.doi.org/10.16250/j.32.1374. 2020263. (In Chinese).

7. Yu JM, De Vlas SJ, Jiang QW, Gryseels B. Comparison of the KatoKatz technique, hatching test and indirect hemagglutination assay (IHA) for the diagnosis of Schistosoma japonicum infection in China. Parasitol Int 2007;56(1):45 - 9. http://dx.doi.org/10.1016/j.parint. 2006.11.002.

8. Qin ZQ, Xu J, Feng T, Lv S, Qian YJ, Zhang LJ, et al. Field evaluation of a loop-mediated isothermal amplification (LAMP) platform for the detection of Schistosoma japonicum infection in Oncomelania hupensis snails. Trop Med Infect Dis 2018;3(4):124. http://dx.doi.org/10.3390/ tropicalmed3040124.

9. Wang LD, Chen HG, Guo JG, Zeng XJ, Hong XL, Xiong JJ, et al. A strategy to control transmission of Schistosoma japonicum in China. N Engl J Med 2009; 360(2): 121-8. https://www.nejm.org/doi/full/10. 1056/NEJMoa0800135.

10. Zhang LJ, Dai SM, Xue JB, Li YL, Lv S, Xu J, et al. The epidemiological status of schistosomiasis in P.R. China after the World Bank Loan Project, 2002-2017. Acta Trop 2019;195:135-41. http://dx.doi.org/10.1016/j.actatropica.2019.04.030 